Revista PSICOLOGIA, 2015, Vol. 29 (2), 1-16. doi: http://dx.doi.org/10.17575/rpsicol.v29i2.1002

\title{
Mas Porquê? Um Estudo Multiteórico dos Preditores da Infidelidade
}

\author{
Thaysa Alessandra Viegas ${ }^{1}$, João Manuel Moreira ${ }^{1}$ \\ ${ }^{1}$ Faculdade de Psicologia da Universidade de Lisboa
}

\begin{abstract}
Resumo: Este estudo pretendeu contribuir para o avanço do conhecimento acerca da infidelidade conjugal, através da identificação dos seus principais preditores, partindo de múltiplas perspetivas teóricas e comparando a sua capacidade preditiva. As variáveis foram avaliadas através de instrumentos de autorrelato, numa amostra de 545 indivíduos da comunidade, recolhida através da Internet. Um ano depois, 53 participantes forneceram novos dados, para avaliação da continuidade dos comportamentos. Verificou-se um predomínio de dois tipos de preditores: (a) a baixa qualidade e investimento na relação primária, e (b) o uso de uma estratégia reprodutiva a curto prazo. 0 primeiro preditor foi mais saliente no sexo feminino e para o envolvimento afetivo, o segundo no sexo masculino e para o envolvimento sexual. Outros preditores significativos surgiram em contextos específicos, mostrando que, embora algumas teorias se tendam a confundir em termos do seu papel preditivo, nenhuma permite por si só compreender o fenómeno do envolvimento extrarrelacional.
\end{abstract}

Palavras-chave: Infidelidade; Psicologia Evolutiva; Vinculação; Conjugalidade; Sexualidade.

But why? A Multitheoretical Study of the Predictors of Infidelity: This study intended to contribute to the advancement of knowledge on marital infidelity, through the identification of its main predictors, from multiple theoretical perspectives and comparing their predictive capacity. Variables were measured by self-report, in a sample of 545 individuals from the community, responding over the Internet. One year later, 53 participants provided further data, to allow for the evaluation of behavior continuity. Two predictors were found to predominate: (a) low quality and investment in the primary relationship and (b) the use of a short-term reproductive strategy. The first predictor was more salient among females and for affective involvement, the second among males and for sexual involvement. Other significant predictors were found in specific contexts, showing that, although some theories tend to be confounded in their predictive capacity, none of them singly allows for a sufficient comprehension of the phenomenon of extra-relational involvement.

Keywords: Infidelity; Evolutionary Psychology; Attachment; Marriage; Sexuality.

Apesar de posta em causa por novos modelos relacionais (Klesse, 2006) a fidelidade parece continuar a ser um aspeto muito valorizado na conjugalidade contemporânea (Treas \& Giesen, 2000). Não obstante esta valorização, a prática clínica e a investigação no campo das relações de casal têm evidenciado que a infidelidade ocorre com elevada prevalência (De Stefano \& Oala, 2008; Duba, Kindsvatter \& Lara, 2008; Goldenberg, 2013; Treas \& Giesen, 2000). Partindo desta constatação, Goldenberg (2008) introduziu o conceito de "fidelidade paradoxal" para ilustrar a aparente importância da fidelidade, mesmo quando ocorre infidelidade: a primeira surgiria assim como um objetivo, mesmo quando há envolvimento em comportamentos extrarrelacionais. Parece, portanto, existir uma larga discrepância entre as normas sociais adotadas pela generalidade dos indivíduos e os seus comportamentos. Esta discrepância não poderá senão derivar de importantes mecanismos motivacionais que conduzam o comportamento em direções discrepantes com os valores assumidos, mecanismos esses que a psicologia deverá, obviamente, tentar estudar e explicar, até pelas importantes consequências negativas que a infidelidade tem para os casais e as famílias (De Stefano \& Oala, 2008; Foster \& Misra, 2013; Hall \& Fincham, 2006).

São várias as teorias que tentam fornecer uma leitura explicativa da ocorrência de infidelidade. Assim, o objetivo deste estudo foi o de confrontar a capacidade que cada uma delas tem para prever a ocorrência de comportamentos de infidelidade, a partir das variáveis antecedentes que postula. Este confronto poderá permitir-nos aquilatar do valor relativo das diferentes teorias nesta previsão, assim como as suas eventuais confluências ou domínios específicos de aplicabilidade. Deste modo, poderá ser

1 Dados de contacto para correspondência: João Manuel Moreira, Faculdade de Psicologia da Universidade de Lisboa, Alameda da Universidade, 1649-013 Lisboa, Portugal. E-mail: joao.moreira@campus.ul.pt. 0 trabalho de Thaysa Alessandra Viegas foi realizado com o apoio da Fundação para a Ciência e a Tecnologia, através de uma Bolsa de Doutoramento com a referência BD/61504/2009. 
melhor avaliada a importância e utilidade que cada uma destas perspetivas teóricas, muitas vezes envolvidas em polémicas entre si (Confer, Easton, Fleischman, Goetz, Lewis, Perilloux, \& Buss, 2010; Eastwick, Luchies, Finkel, \& Hunt, 2014; Gilding, 2009; Hazan \& Diamond, 2000; Smiler, 2011), poderá ter na compreensão do fenómeno da infidelidade e na intervenção sobre ele.

A psicologia evolutiva, que tem por base o conceito de evolução no seu sentido biológico, proposto nos seus fundamentos por Darwin (Buss, 2005, 2008), sugere que os mecanismos psicológicos resultam de processos de seleção natural. Ao longo de muitas gerações, os genes que dão origem a predisposições psicológicas associadas a um maior sucesso em termos reprodutivos deverão tornar-se predominantes na população humana. As preferências percetivas, motivacionais e comportamentais observadas nos seres humanos atuais refletiriam, deste modo, os problemas adaptativos com que a espécie se defrontou no passado: para além das necessidades de sobrevivência, a obtenção de parceiros reprodutivos e o assegurar da sobrevivência e sucesso reprodutivo dos descendentes (Buss, 2008).

Ora, neste propósito de maximização do sucesso reprodutivo, os problemas confrontados por homens e mulheres são bastante diferentes. Esta discrepância deriva, sobretudo, das diferenças no investimento parental, inevitavelmente superior nas mulheres, quer a nível do tempo, quer dos riscos, quer dos recursos despendidos (gravidez, lactação). Assim, prevê-se que diferenças de género nos mecanismos psicológicos ligados ao acasalamento e reprodução surjam nos domínios em que os dois sexos enfrentaram recorrentemente diferentes problemas adaptativos, ao longo da história evolutiva (Buss, 2008). Sendo o investimento parental mínimo muito reduzido, no caso dos homens, estes terão vantagem em tentar assegurar o maior número possível de descendentes, reduzindo o seu nível de investimento parental. Nas mulheres, cujo número máximo de descendentes é muito limitado, a maior preocupação será com a sobrevivência e o sucesso competitivo destes, o que deverá levar a um maior investimento parental, procurando um companheiro que seja também cuidador dos filhos. Por isso, as mulheres tenderão a adotar uma orientação mais emocional e interpessoal para a sexualidade, com foco nos fatores interpessoais, enquanto os homens terão uma postura mais recreativa, enfatizando a necessidade de exprimir os seus desejos sexuais, com as suas fantasias a tenderem a ser mais explícitas, com desejo de variedade e gratificação física (Ellis \& Symons, 1990).

Buss e Schmitt (1993) introduziram o conceito de estratégias sexuais a curto e a longo prazo para ilustrar esta diferença entre os sexos. No entanto, embora a estratégia a curto prazo (baseada na quantidade) seja mais saliente nos homens e a estratégia a longo prazo (baseada na qualidade) seja mais saliente nas mulheres, diferenças de grau na utilização destas estratégias existem mesmo dentro de cada sexo. 0 facto de o comportamento dos indivíduos refletir a ponderação relativa destas duas estratégias resulta com certeza de a sua eficácia depender de variáveis contextuais. Assim, por exemplo, uma estratégia a longo prazo, com um forte investimento numa relação de casal duradoura e nos cuidados parentais a um número reduzido de descendentes, poderá ser desvantajosa se estas relações estiverem sujeitas a um elevado risco, seja por efeito de uma elevada taxa de mortalidade, seja pela falta de confiança na reciprocidade do investimento por parte do parceiro. Pelo contrário, num contexto de baixa mortalidade, onde a competição intraespecífica prevalece sobre a sobrevivência, o investimento parental intenso pode ser essencial para habilitar a descendência para essa competição (Belsky, 1999; Kirkpatrick, 1998).

A teoria das estratégias sexuais propõe-se explicar também as diferenças encontradas entre homens e mulheres no que concerne à infidelidade. Assim, indivíduos que utilizem em maior grau uma estratégia a curto prazo, tendendo a procurar maior número e variedade de parceiros sexuais, terão maior probabilidade de ser infiéis, de expressar esse desejo e ter maior apetência para um envolvimento sexual. Esta situação deverá ser, portanto, mais comum entre os homens. Pelo contrário, aqueles que seguem uma estratégia a longo prazo terão menor propensão para a infidelidade e, se esta acontecer, tenderá a caracterizar-se por um maior envolvimento emocional e a ser em maior grau determinada pela procura de um parceiro mais capaz ou mais disposto a investir recursos. Esta tendência deverá ser mais saliente entre as mulheres (Barta \& Kiene, 2005; De Stefano \& Oala, 2008).

A teoria da vinculação (Hazan \& Diamond, 2000; Hazan \& Shaver, 1994) é outra abordagem potencialmente útil na explicação das diferenças individuais na sexualidade. A teoria postula que os seres humanos possuem uma predisposição inata, desde a infância, para estabelecer relações significativas que lhes proporcionem segurança. Na idade adulta, essa função é predominantemente desempenhada pelas relações de casal, nas quais os sistemas de vinculação, prestação de cuidados e acasalamento se interligam, funcionando em conjunto e influenciando-se mutuamente (Hazan \& Diamond, 2000). Assim, embora o sistema de vinculação e o sistema sexual sejam distintos, com funções diferentes, as diferenças individuais no estilo de vinculação têm uma importante influência no comportamento sexual (Fisher, Aron, Mashek, Li, \& Brown, 2002). 
O estilo seguro é caraterístico de indivíduos confiantes no seu parceiro e na sua responsividade, que sentem prazer com a proximidade do outro e com o desenvolvimento de atividade sexual no contexto de uma relação longa. As suas relações tendem a ser bem-sucedidas e com menor probabilidade de dissolução. 0 estilo de vinculação ansioso/ambivalente ou preocupado configura um padrão em que coexistem um forte desejo de proximidade e uma falta de confiança na resposta do parceiro, resultando em necessidades de vinculação excessivas e em sentimentos contraditórios, não só durante episódios negativos mas também durante aqueles que supostamente seriam positivos. Uma expectativa negativa generalizada em relação à responsividade emocional por parte dos outros resulta no estilo evitante, com aversão pelo contacto emocional íntimo, um menosprezo pelo investimento nas relações e uma maior valorização da independência e da autossuficiência (Mikulincer \& Shaver, 2005; Zeifman \& Hazan, 1997).

Estes estilos de vinculação têm implicações para o comportamento sexual, eventualmente diferenciadas em função do género (Hazan \& Diamond, 2000; Hazan \& Shaver, 1994). De facto, o sexo tem o potencial de motivar experiências intensas e prazerosas, promovendo ligações significativas entre parceiros românticos, mas pode também ser uma das causas do afastamento entre esses mesmos parceiros: pode ser propiciador de validação ou de baixa autoestima (Birnbaum, Mikulincer, Szepsenwol, Shaver \& Mizrahi, 2014). Indivíduos ansiosos/ambivalentes tenderão a usar o sexo como forma de tentar preencher as suas necessidades de vinculação, relacionadas com segurança e amor. A literatura mostra, com efeito, que as mulheres ansiosas/ambivalentes têm tendência a manter uma atividade sexual intensa e comportamentos de risco, enquanto os homens com o mesmo estilo terão uma expressão sexual mais restrita (Stefanou \& McCabe, 2012). Por outro lado, a atividade sexual, envolvendo intimidade física e emocional, pode criar desconforto em pessoas com um estilo de vinculação evitante, que habitualmente mantêm certa distância física e emocional do parceiro. Não surpreende, portanto, que a investigação tenha encontrado nestas pessoas uma tendência para a aversão ao contacto emocional íntimo, como beijos e carícias, e preferência por contacto sexual puro, vaginal, oral e anal (Zeifman \& Hazan, 1997), em relações menos duradouras e com menor compromisso (Stefanou \& McCabe, 2012).

Nesta perspetiva, as diferenças individuais no funcionamento do sistema sexual podem ser entendidas em termos de hiperativação ansiosa ou desativação evitante do sistema de vinculação (Birnbaum et al., 2014). Em qualquer caso, os dados disponíveis sugerem que os estilos de vinculação inseguros parecem estar associados a um conjunto de comportamentos (maior número de parceiros, mais relações ocasionais) que configuram uma estratégia sexual a curto prazo, enquanto o estilo de vinculação seguro estará interligado com comportamentos que se podem associar a uma estratégia de longo prazo (maior investimento numa relação estável; Stefanou \& McCabe, 2012). Outro conjunto de dados associa diferentes estilos de vinculação à qualidade da relação de casal, com o estilo evitante associado a maior insatisfação e maior probabilidade de divórcio (Mikulincer, Florian, Cowan \& Cowan, 2002; Moreira et al., 2006). É plausível que estes efeitos sejam produto das diferentes estratégias sexuais associadas aos estilos de vinculação.

A presença destas relações, entretanto, e sabendo-se como o estilo de vinculação nos adultos é o reflexo das experiências de infância com os prestadores de cuidados (Hazan \& Shaver, 1994), permite levantar a hipótese de que o estilo de vinculação funcione como mediador na relação entre as experiências precoces e as estratégias sexuais (Belsky, Steinberg, \& Draper, 1991; Kirkpatrick, 1998; Leal, 2009). Pressupondo que as estratégias sexuais serão adaptativas, no sentido evolutivo, por constituírem uma resposta ao ambiente ecológico (Schmitt, 2005a), seria plausível pensar que o estilo de vinculação consiste num mecanismo ontogenético de adaptação ao ambiente social expectável.

Esta hipótese foi já apoiada por alguns dados, que se procurarão aprofundar e confirmar nesta investigação. Por exemplo, Moreira (2009) evidenciou que a aceitação pelos pais na infância, assim como, nos homens, o encorajamento da sua independência, se relacionam de modo negativo com a dimensão evitação do estilo de vinculação adulta. Por seu turno, a evitação está associada a uma maior disponibilidade para a utilização de estratégias de curto prazo, enquanto a preocupação se associa negativamente a esta estratégia sexual.

Outra literatura (Belsky, 1999; Belsky, Steinberg \& Draper, 1991; Ellis, Bates, Dodge, Fergusson, Horwood, Pettit \& Woodward, 2003; Schmitt, 2005b; Simpson \& Gangestad, 1991; Vigil \& Geary, 2006) sugere uma relação entre as experiências familiares precoces e as estratégias sexuais, mas não parecem totalmente esclarecidos quais os fatores das primeiras que atuam como determinantes das segundas. Belsky, Steinberg e Draper (1991) verificaram que as experiências familiares negativas na pré-puberdade aceleram o aparecimento da puberdade, favorecem uma sexualidade mais precoce e a formação de laços menos sólidos nas relações de casal. Draper e Harpending (1982) alertam para a influência determinante da ausência do pai durante a infância, com efeitos diferentes consoante o género dos filhos. Assim, os rapazes adotariam comportamentos exageradamente masculinos e estereotipados na infância, e as mulheres tenderiam a exibir comportamentos de promiscuidade sexual na adolescência. Leal (2009) 
concluiu que, nas mulheres, o stress familiar é a variável das experiências de infância que se destaca, causando dois efeitos aparentemente opostos: um aumento do compromisso, através do aumento da preocupação, e uma diminuição do compromisso através de um aumento das estratégias de curto prazo, que por sua vez vão diminuir a satisfação. Foram encontrados efeitos diferentes no homem, sendo neste caso a qualidade da relação com a mãe que assume um papel preponderante nas experiências de infância, fazendo diminuir a evitação e, com ela, o uso das estratégias de curto prazo. Numa perspetiva psicanalítica, Josephs (2006) defende que a infidelidade pode ser uma consequência de temáticas edipianas mal resolvidas, em que o progenitor desejado, do sexo oposto, é visto como cometendo uma infidelidade com o progenitor do mesmo sexo. Se não for adequadamente resolvida, esta ferida narcísica vai resultar em comportamentos de infidelidade na vida adulta, sentidos como uma vingança pela infidelidade atribuída ao objeto amado primordial. Entretanto, Poulsen, Holman, Busby e Carrol (2013) concluíram que quer a atração física quer os estilos de vinculação desempenham um papel importante no início de uma relação amorosa. Assim, defendem que ambas as teorias (estratégias sexuais e vinculação) serão importantes na escolha de um parceiro e na manutenção de uma relação, donde se pode deduzir que ambas desempenharão um papel na origem dos comportamentos de infidelidade.

Muitos outros fatores, no entanto, poderiam ser apontados como influenciando a probabilidade de envolvimento em comportamentos extrarrelacionais. Por exemplo, Brooks e Monaco (2013) sugerem que as diferenças entre homens e mulheres estarão relacionadas com diferentes objetivos associados à reprodução e à descendência. Estes autores defendem que uma relação estável com um parceiro primário com caraterísticas semelhantes à do próprio diminui a probabilidade de envolvimento extraconjugal. Por outro lado, concluíram que estar empregado e manter uma relação conjugal com alguém que não trabalha aumenta a probabilidade de envolvimento sexual extraconjugal para ambos os géneros. Elmslie e Tebaldi (2008) reforçam a ideia que os fatores económicos interagem com os aspetos biológicos do comportamento humano. Assim, homens e mulheres avaliarão de modo diferente o custo/benefício da infidelidade, com a classe social de pertença, o grau de religiosidade, a dimensão da localidade e a educação a terem impacto no comportamento da mulher, e a educação, a felicidade e a área rural/urbana de habitação, assim como o estatuto social relativo ao emprego, a influenciar o homem. Por outro lado, e ainda segundo os mesmo autores, o comportamento extraconjugal dos homens parece ser determinado pelas suas próprias caraterísticas e não pelas da parceira, contrariamente ao que parece verificar-se para as mulheres, em que a infidelidade está associada às caraterísticas da relação primária e do parceiro. Finalmente, nas mulheres, a probabilidade de envolvimento extraconjugal parece aumentar com a fertilidade, decrescendo os benefícios do primeiro quando a segunda diminui.

Outro fator suscetível de influenciar o envolvimento em comportamentos extrarrelacionais é a atitude dos indivíduos em relação a estes. Considerou-se importante incluir a avaliação da atitude face à infidelidade, dado que a literatura indica não só que as atitudes podem ser um importante preditor do comportamento neste domínio, como também que apresentam diferenças significativas entre sexos (Afonso, 2011; Glass \& Wright, 1992; Wilson, Mattingly, Clark, Weidler \& Bequette, 2011), podendo ajudar a explicar as eventuais diferenças no comportamento extrarrelacional de homens e mulheres.

Para além dos fatores que poderão predispor em diferente grau os indivíduos para a infidelidade, parece importante também olhar para o lado positivo e para os fatores que predispõem para a fidelidade. Diferentes tipos de motivações para a fidelidade poderão ter impactos diferentes nos comportamentos extrarrelacionais. Nomeadamente, a motivação intrínseca está associada a comportamentos iniciados pelo prazer e satisfação que proporcionam, que têm um carácter voluntário e não estão dependentes de uma recompensa material, enquanto a motivação extrínseca engloba comportamentos iniciados por razões instrumentais, como receber uma recompensa ou evitar uma punição (Pelletier, Tuson, Haddad, 1997). De acordo com a teoria da autodeterminação (Deci \& Ryan, 2008), as motivações diferenciam-se num contínuo entre a motivação regulada mais externamente (extrínseca) e mais internamente (intrínseca). Assim, a regulação externa corresponde aos comportamentos controlados por fontes externas, como recompensa material ou constrangimento imposto por outra pessoa. A regulação introjetada verifica-se quando a fonte externa de motivação já foi parcialmente internalizada e já não é necessária a sua presença para a pessoa iniciar o comportamento. Este será reforçado por pressões internas como a culpa, a ansiedade e emoções relacionadas com a autoestima. Na regulação identificada, o comportamento é iniciado porque é congruente com valores e objetivos pessoais. Por fim, na regulação integrada, o comportamento é valorizado pelo seu significado e por ser consistente com a identidade do indivíduo. Esta última será a motivação mais autodeterminada dentro deste grupo de motivações que, em todos os casos, mantêm um componente de regulação extrínseca. Acima deles, a motivação intrínseca corresponde ao mais elevado grau de regulação interna. Fora deste contínuo, a ausência de motivação (amotivação) surgirá quando o sujeito não percebe a relação entre o comportamento e os resultados do 
mesmo, e experimenta sentimentos de incompetência e ausência de controlo, iniciando uma atividade sem ter um entendimento sobre as razões pelas quais o está a fazer (Deci \& Ryan, 2008).

Hipotetiza-se que a presença de uma motivação internalizada terá consequências positivas para a relação amorosa (Leak \& Cooney, 2001). Esta, porém, parece ser específica em face do contexto, ou seja, um indivíduo pode ter um determinado tipo de motivação para se manter numa relação, mas a motivação para a realização de diversas atividades com o parceiro pode ser diferente. De facto, Gaine e La Guardia (2009), na sua investigação, concluíram que é fundamental avaliar não só a motivação para que alguém permaneça numa relação, mas também a motivação para a realização de várias atividades com o parceiro, dado que estas também se afiguram importantes para que a relação funcione. Assim, o estudo das motivações para a fidelidade parece poder constituir um objetivo importante para a investigação nesta área.

0 presente estudo tem, assim, como objetivo contribuir para um melhor conhecimento sobre os preditores da infidelidade. Persegue esse objetivo colocando em confronto, no mesmo estudo, um conjunto de variáveis propostas por diferentes perspetivas teóricas. Dado que essas perspetivas teóricas se apresentam como candidatas alternativas à predição e explicação dos comportamentos extrarrelacionais, importa perceber qual o contributo que cada uma é efetivamente capaz de dar, em confronto competitivo com outras. Não é também de excluir a possibilidade de o possível contributo de algumas delas poder afinal ser esvaziado pelo poder preditivo, e talvez explicativo, de outras. 0 confronto empírico é essencial para dirimir entre explicações teóricas rivais, e o presente estudo procura justamente provocar e analisar esse confronto.

\section{MÉTODO}

\section{Participantes}

Participaram neste estudo 545 indivíduos, 149 homens (27.5\%) e 396 mulheres (72.5\%). A média de idades dos participantes no primeiro momento de recolha de dados foi de 30.01 anos $(D P=10.06)$, com idades compreendidas entre os 18 e os 70 anos. No que concerne à escolaridade, $69 \%$ possuíam habilitações ao nível do ensino superior e $28 \%$ do ensino secundário. Somente dois participantes tinham escolaridade até 6 anos. Havia 25\% de sujeitos casados, 1\% estavam noivos, 15\% viviam em união estável, $31 \%$ estavam numa relação de namoro, $22 \%$ não estavam envolvidos em nenhuma relação e $4 \%$ estavam divorciados ou separados. A duração média da relação foi de 8.71 anos ( $D P=7.47)$. Uma percentagem elevada (71\%) declarou não ter tido casamentos ou uniões anteriores, $18 \%$ terá tido uma relação ou união anterior. No que diz respeito à classe socioeconómica atual, $59 \%$ consideraram -se inseridos na classe média, $14 \%$ na classe média alta e $24 \%$ na classe média baixa, sendo as respostas semelhantes quando perguntado acerca da classe socioeconómica em que cresceram. Os católicos eram $52 \%$, enquanto $37 \%$ afirmaram não ter nenhuma religião. Quanto ao grau de religiosidade, $78 \%$ situavam-se entre o nada religioso e um nível moderado de religiosidade. Dentro da orientação política, $60 \%$ dos participantes considerava-se de direita, $17 \%$ ligeiramente à direita, $17 \%$ de centro e $6 \%$ de esquerda. Na segunda fase do estudo participaram 53 pessoas, 7 homens (13.2\%) e 46 mulheres (86.8\%).

\section{Instrumentos}

\section{Infidelidade}

Foi utilizado o "Questionário Multidimensional de Comportamentos Extra-Relacionais" (QMCER), desenvolvido no âmbito desta investigação, com o objetivo de caraterizar múltiplos aspetos como comportamentos, motivações e contextos da ocorrência de infidelidade. Este instrumento é descrito em detalhe em Viegas e Moreira (2014). No presente estudo foram utilizadas as suas secções C, D e F.

Na secção C são apresentados diversos comportamentos, com o intuito de avaliar se o sujeito realizou alguns deles com pessoas que não o seu parceiro, numa altura em que estava envolvido numa relação amorosa com compromisso de exclusividade, atual ou passada. Procurou-se, na elaboração desta secção, incluir uma variedade de comportamentos concretos e específicos, passíveis de ocorrer numa situação de infidelidade e que representassem diferentes tipos e graus de envolvimento físico, emocional e sexual. No aspeto que vai ser utilizado neste estudo, os 10 itens eram respondidos em termos de (a) "Não aconteceu" / (b) "Aconteceu". Na secção D são apresentados de modo hipotético os mesmos itens da secção C. Esta secção destina-se apenas aos indivíduos que responderam nunca ter estado envolvidos numa relação amorosa com compromisso de exclusividade. Para todos os outros, esta secção é omitida. Optou-se por agregar, na análise, as secções $\mathrm{C}$ e $\mathrm{D}$, dado que o número de sujeitos que responderam à secção D era muito reduzido $(n=32)$ e a análise apenas da secção $C$ fornecia resultados praticamente idênticos.

Foi efetuada uma análise em componentes principais ao nível dos itens das secções C e D, com os dados do primeiro momento de recolha de dados, uma vez que o número de participantes no segundo 
momento foi muito mais reduzido. Foi utilizado o scree plot (Moreira, 2004) como critério para a determinação do número de fatores, e uma rotação do tipo Varimax. 0 índice de Kaiser-Meyer-Olkin e o teste de esfericidade de Bartlett foram calculados e mostraram resultados adequados em todos os casos, não sendo aqui apresentados em detalhe por razões de espaço. Os três fatores encontrados pareciam estar associados ao envolvimento físico (Fator 1; item exemplo: "Manter relações sexuais com outra pessoa"), ao envolvimento afetivo (Fator 2: "Dizer 'amo-te' a outra pessoa") e ao desejo sexual (Fator 3: "Sentir-se sexualmente atraído por outra pessoa"). Foram derivadas destes fatores escalas compostas pelos itens com saturações superiores a .50 na matriz após rotação. Este valor, utilizado de forma sistemática nas diferentes escalas, permitiu alcançar um bom compromisso em termos da especificidade do conteúdo das escalas e dos valores do alfa de Cronbach. A escala de Envolvimento Físico, composta por quatro itens, obteve um alfa de Cronbach de ,88, a de Envolvimento Afetivo, também com quatro itens, um alfa de ,78 e a de Desejo sexual, com dois itens, um alfa de .81 .

A secção F (13 itens) pretende avaliar as diferentes motivações para o não envolvimento em comportamentos extrarrelacionais, ou seja, as motivações para a fidelidade. A escala vai de 1, "nada verdadeira" a 7, "completamente verdadeira". É preenchida por todos os participantes. A análise em componentes principais, realizada de modo semelhante ao anteriormente descrito, conduziu à identificação de quatro fatores. 0 Fator 1 parece corresponder à motivação intrínseca para o não envolvimento extrarrelacional (item exemplo, "Amar o seu parceiro"), o Fator 2 à motivação introjectada ("Condenar a traição"), o Fator 3 à motivação extrínseca ("Ter medo de ser descoberto"), e o fator 4 à amotivação ("Não conhecer ninguém que lhe desperte interesse"). A escala de Motivação Intrínseca (3 itens) obteve um alfa de Cronbach de .83, a de Motivação Introjectada (4 itens) de .97, a de Motivação Extrínseca (3 itens) de .70 e a de Amotivação (2 itens) de .50. Apesar do valor insuficiente do coeficiente alfa de Cronbach para o último fator, optámos por mantê-lo, dado que corresponde a uma dimensão frequentemente encontrada em análises fatoriais de questionários de motivação e poderá ser alvo de futuros desenvolvimentos, com a adição de novos itens que aumentem a sua precisão. Na forma atual do questionário, não havia mais itens disponíveis que ajudassem a atingir este objetivo.

\section{Atitudes em relação à Infidelidade}

Foi utilizada a Escala de Atitudes em Relação à Infidelidade desenvolvida por Afonso (2011). Efetuou-se uma reanálise dos dados do estudo de Afonso (2011), tendo sido selecionados os quatro itens com maior saturação, por forma a obter uma versão breve da escala (exemplo de item: "A infidelidade é um modo de vida normal que não tem nada de reprovável”). É pedido aos participantes que indiquem o seu grau de concordância com as frases, numa escala de 1, "discordo fortemente" a 5, "concordo fortemente". 0 alfa de Cronbach obtido foi de.73.

\section{Satisfação Conjugal}

Foi utilizada uma versão reduzida da Relationship Rating Form (RRF), versão portuguesa de Lind (2008), com os 33 itens mais representativos dos fatores identificados nesta versão. Neste questionário é pedido aos sujeitos que classifiquem cada um dos itens numa escala de 9 pontos, de 1 ("nada") a 9 ("completamente ou extremamente"). Optou-se pela realização de uma análise em componentes principais para confirmar a estrutura fatorial da escala, utilizando as mesmas técnicas já descritas anteriormente. Assim, podemos dizer que o Fator 1 corresponde à Confiança (item exemplo: "Esta pessoa pode contar consigo para a ajudar quando precisar?"), o Fator 2 à Paixão (item exemplo: "Dá-lhe prazer, só de observar ou olhar para esta pessoa?") e o Fator 3 ao Conflito (item exemplo: "Você briga e discute com esta pessoa?") No presente estudo, o alfa obtido foi de .94 na escala de Confiança, de .93 na escala de Paixão e de .89 na escala de Conflito. Cada escala era constituída por 9 itens.

\section{Estilo de Vinculação Adulta}

O questionário "Experiências em Relações Próximas (ERP)" é a versão portuguesa do "Experiences in Close Relatioships" (Brennan, Clark \& Shaver, 1998), traduzido e adaptado por Moreira et al. (2006). Procura avaliar as duas dimensões básicas das diferenças individuais no estilo de vinculação dos adultos: a Evitação e a Preocupação. A forma reduzida utilizada neste estudo é composta por 12 itens, com uma escala de avaliação de sete pontos que vai de 1, "discordo totalmente" a 7, "concordo fortemente", dos quais seis medem a Evitação (item exemplo: "Não me sinto confortável ao 'abrir-me' com o meu parceiro/a") e 6 a Preocupação (item exemplo: "Fico ressentido/a quando o meu parceiro/a passa tempo longe de mim"). Foi efetuada uma análise em componentes principais ao nível dos itens, que confirmou a sua distribuição pelos dois fatores, tendo a escala de Evitação obtido um alfa de .75 e a de Preocupação um alfa de.78. 
Estratégias Sexuais

O Questionário de Estratégias Sexuais (QES), desenvolvido por Moreira (2009), é constituído por um total de 12 itens, divididos por duas escalas que pretendem medir a utilização das estratégias a longo prazo e da estratégia a curto prazo. Em função dos resultados obtidos em versões anteriores, foi decidido elaborar uma nova versão do QES, que se distingue da anterior por separar as referências à dedicação ao companheiro/a e aos filhos em itens diferentes. Assim, o QES-II ficou constituído por 13 itens, que pretendem medir a utilização de estratégias a curto e a longo prazo, sendo que na segunda se procura agora diferenciar entre o investimento no companheiro/a e nos filhos. Os sujeitos deverão indicar o grau em que cada um dos itens corresponde aos seus comportamentos, sentimentos e atitudes, através de uma escala de Likert de 7 pontos que vai de 1, "Não tem a ver comigo" a 7, "tem totalmente a ver comigo".

Uma análise em componentes principais permitiu a identificação de três fatores. Podemos dizer que o Fator 1 (5 itens) corresponde à estratégia a curto prazo (item exemplo: "Sou infiel por natureza"), o Fator 2 (5 itens) à estratégia a longo prazo com investimento no parceiro (item exemplo: "Quero que o meu marido/esposa ou companheiro/a seja o aspeto central da minha vida") e o Fator 3 (3 itens) à estratégia a longo prazo com investimento nos filhos (item exemplo: "Tenho a intenção de investir muito tempo e esforço na relação com os meus filhos"). Refira-se que os itens 7 e 11, ambos referentes à família em geral, saturaram predominantemente no fator correspondente à estratégia a longo prazo com investimento no parceiro. 0 alfa obtido foi de .84 para a escala Estratégia a Curto Prazo; de .77 para a escala Estratégia a Longo Prazo com Investimento no Parceiro e de .78 para a Estratégia a Longo Prazo com Investimento nos Filhos.

\section{Orientação Sociosexual}

O Inventário de Orientação Sociosexual (IOSS; Simpson \& Gangestad, 1991) foi construído tendo como objetivo fornecer uma medida global da predisposição dos indivíduos para se envolverem em relações sexuais casuais, sem compromisso. A versão portuguesa utilizada neste estudo foi elaborada pela equipa que recolheu em Portugal os dados para o International Sexuality Description Study (Moreira, Mata, Veríssimo, Mata \& Moreira, 2003). É composto por sete itens, dos quais quatro se referem a comportamentos sexuais envolvendo diferentes parceiros: dois referem-se a comportamentos passados (número de parceiros sexuais no último ano e número de parceiros com quem teve relações sexuais uma única vez), um a expectativas de comportamentos futuros (número de parceiros sexuais esperados nos próximos cinco anos) e outro a fantasias sexuais (frequência, numa escala não linear). Os restantes três itens referem-se a atitudes relativas ao sexo casual, avaliadas em escalas de concordância com nove pontos. A análise em componentes principais (seguindo o procedimento já descrito) permitiu verificar que os itens relativos a comportamentos e os relativos a atitudes se separavam claramente em dois fatores distintos. Em virtude dos diferentes formatos de resposta, foi necessário proceder à padronização dos itens para se poder efetuar o cálculo do alfa de Cronbach. Neste cálculo, verificou-se que os itens referentes a expectativas de comportamentos futuros e a fantasias eram indicadores fracos do fator de Comportamentos. A sua eliminação permitiu aumentar o valor do coeficiente alfa para este fator. Assim, os valores finais do coeficiente alfa foram de .88 para a escala de Comportamentos e de .80 para a escala de Atitudes.

\section{Experiências Precoces}

Foi utilizado o Questionário de Experiências de Infância, QEI (Leal, 2009), que foi elaborado com o objetivo de permitir uma avaliação das experiências de infância. Este questionário é composto por uma primeira parte que avalia o número de anos de presença da mãe, do pai, de uma madrasta e de um padrasto nos primeiros dezasseis anos de vida e por duas secções com itens bipolares avaliando o nível de stress na vida familiar na infância e na adolescência (dois itens, e.g., "stressante vs. tranquila") e a qualidade da relação entre os pais (dois itens, e.g., "muito infeliz vs muito feliz"). Foram também utilizados 6 itens da Mother-Father-Peer-Scale (MFPS), versão portuguesa de Lopes dos Santos, Silva e Sousa (1995), que avalia a qualidade das relações com os pais na infância; 3 dos itens são relativos à relação com a mãe e 3 à relação com o pai. 0 alfa de Cronbach foi de .92 para a escala da relação com a mãe, de .91 para a escala da relação com o pai, de .84 para a escala de qualidade na vida familiar e de .84 para a escala da relação entre os pais. 


\section{Procedimentos}

O estudo combinou análises de caráter transversal e longitudinal, tendo sido recolhidos dados em dois momentos no tempo. No primeiro momento, foram recolhidos dados para todas as variáveis. No segundo momento, um ano depois, os participantes foram novamente contatados, tendo sido avaliada a ocorrência de infidelidade, a satisfação e a estabilidade do casal (se este permanece junto). As análises longitudinais apresentadas visaram perceber os efeitos das variáveis experiências precoces, vinculação e estratégias sexuais sobre as mudanças ocorridas no nível de envolvimento extrarrelacional entre um momento e outro. 0 intervalo de tempo de um ano entre os dois momentos de recolha de dados justifica-se com a necessidade de um período temporal suficiente, que permita verificar efeitos da situação de infidelidade na qualidade da relação de casal, mas não demasiado longo, o que permitiria que outros fatores atuassem de forma mais evidente na relação.

Os dados foram recolhidos na Internet, tendo os questionários sido divulgados nas redes sociais, em sites e blogues na área da psicologia, seguindo depois por amostragem "bola de neve". A participação dos sujeitos foi voluntária, tendo os mesmos sido informados do teor dos questionários a preencher, duração prevista da participação, confidencialidade dos dados e obrigatoriedade de ter 18 anos ou mais para poder participar. Era explicitado que, ao clicarem para prosseguir, davam o seu consentimento e garantiam que eram maiores de 18 anos. Os participantes que declararam nunca ter estado envolvidos numa relação com compromisso de exclusividade, não respondiam à RRF, nem à ERP.

0 método de recolha de dados do segundo momento foi idêntico ao primeiro, tendo o questionário sido enviado por email aos participantes que, no primeiro momento, tinham sinalizado a sua disponibilidade para voltar a participar. Infelizmente, o número de participantes sofreu uma grande redução do primeiro para o segundo momento. 0 caráter sensível da informação recolhida e a necessidade de solicitar informação que permitisse associar os dados dos dois momentos, ainda que sem pôr em causa o anonimato (e.g., aniversário da mãe), impediu que se apresentasse à partida o estudo como envolvendo dois momentos. 0 segundo momento foi apresentado como uma participação adicional voluntária, o que terá contribuído para a elevada perda de participantes e limitou as possibilidades da análise longitudinal. A questão da confidencialidade assumiu particular importância, dada a temática em questão e os potenciais riscos para os participantes, pelo que foi garantido aos mesmos que as respostas aos questionários seriam confidenciais e que seria preservado o seu anonimato, não existindo forma de relacionar um participante com as respostas fornecidas. A investigação teve a aprovação da Comissão de Deontologia da Faculdade de Psicologia da Universidade de Lisboa.

A análise dos resultados foi organizada em três partes. Na primeira, utilizando a amostra, mais numerosa, de participantes no primeiro momento, foi realizada uma análise exploratória, com o objetivo de pré-organizar as variáveis potencialmente preditoras da infidelidade, reduzindo o número de preditores para as análises de regressão subsequentes e a sua multicolinearidade. Na segunda parte, ainda recorrendo apenas aos dados do primeiro momento, foram realizadas análises de regressão multivariadas prevendo as diversas facetas dos comportamentos de infidelidade a partir dos preditores previamente definidos. Na terceira parte, utilizaram -se os dados dos dois momentos para analisar a questão dos preditores das mudanças nos comportamentos de infidelidade, do primeiro para o segundo momento.

\section{RESULTADOS}

\section{Análise em Componentes Principais dos Potenciais Preditores}

Optou-se por efetuar uma análise em componentes principais das variáveis potencialmente preditivas da ocorrência da infidelidade, à luz das diferentes teorias mencionadas. A conveniência desta análise resultou de se terem verificado correlações importantes entre algumas delas, bem como do seu número elevado, que iria tornar a análise demasiado complexa. Foi utilizado o scree plot como critério para a determinação do número de fatores, e uma rotação do tipo Varimax. 0 scree plot indicou cinco fatores, sendo a lista das variáveis com a respetiva saturação em cada fator na matriz rodada apresentada no Quadro 1. 
Quadro 1. Matriz de Saturações dos Potenciais Preditores após Rotação.

\begin{tabular}{|c|c|c|c|c|c|}
\hline \multirow[b]{2}{*}{ Variáveis } & \multicolumn{5}{|c|}{ Fatores } \\
\hline & $\begin{array}{l}\text { Qualidade } \\
\text { relação }\end{array}$ & $\begin{array}{c}\text { Atitudes e curto } \\
\text { prazo }\end{array}$ & $\begin{array}{c}\text { Família de } \\
\text { origem }\end{array}$ & Preocup. & Idade \\
\hline Idade & -0.09 & 0.05 & 0.02 & -0.01 & 0.90 \\
\hline Escolaridade & -0.04 & 0.06 & -0.06 & -0.34 & 0.05 \\
\hline Classe social & 0.00 & -0.18 & 0.25 & -0.39 & -0.16 \\
\hline Duração da relação atual & -0.02 & -0.09 & 0.04 & 0.04 & 0.87 \\
\hline Grau de religiosidade & -0.08 & -0.39 & 0.01 & 0.03 & 0.16 \\
\hline Orientação política & 0.72 & 0.03 & -0.03 & -0.03 & -0.12 \\
\hline Motiv. autónoma & 0.65 & -0.42 & 0.09 & 0.12 & -0.13 \\
\hline Motiv. controlada & 0.22 & -0.56 & 0.06 & 0.42 & -0.24 \\
\hline Motiv. extrínseca & -0.30 & 0.25 & 0.01 & 0.43 & -0.19 \\
\hline Amotivação & -0.00 & -0.20 & 0.09 & 0.17 & 0.02 \\
\hline Atitudes Infidelidade & -0.22 & 0.73 & -0.08 & -0.19 & 0.13 \\
\hline Confiança & 0.82 & -0.06 & 0.15 & 0.10 & 0.09 \\
\hline Paixão & 0.78 & -0.11 & 0.07 & 0.13 & -0.11 \\
\hline Conflito & -0.71 & 0.24 & -0.11 & 0.25 & -0.11 \\
\hline Evitação & -0.83 & 0.12 & 0.02 & 0.06 & 0.12 \\
\hline Preocupação & -0.01 & -0.08 & -0.06 & 0.65 & -0.17 \\
\hline Estratégia a curto prazo & -0.34 & 0.81 & -0.03 & 0.07 & 0.08 \\
\hline Estr. longo prazo parceiro & 0.52 & -0.22 & 0.13 & 0.56 & 0.07 \\
\hline Estratégia a curto prazo & -0.34 & 0.81 & -0.03 & 0.07 & 0.08 \\
\hline Estr. longo prazo parceiro & 0.52 & -0.22 & 0.13 & 0.56 & 0.07 \\
\hline Estr. longo prazo parental & 0.07 & -0.09 & 0.07 & 0.51 & 0.21 \\
\hline Comportamento sexual & -0.07 & 0.44 & -0.08 & 0.26 & -0.07 \\
\hline Atitudes sexo casual & -0.01 & 0.76 & -0.03 & -0.06 & -0.03 \\
\hline Presença de madrasta & 0.13 & -0.00 & -0.05 & 0.04 & 0.02 \\
\hline Presença da mãe & -0.03 & -0.14 & 0.30 & -0.15 & -0.05 \\
\hline Presença de padrasto & 0.24 & 0.12 & -0.28 & -0.08 & -0.07 \\
\hline Presença do pai & -0.11 & -0.11 & 0.57 & 0.05 & 0.15 \\
\hline Qualidade relação mãe & 0.15 & -0.10 & 0.74 & 0.08 & -0.02 \\
\hline Qualidade relação pai & 0.16 & 0.05 & 0.58 & 0.21 & -0.15 \\
\hline Qualidade relação pais & -0.01 & 0.01 & 0.79 & -0.05 & 0.06 \\
\hline Qualidade vida familiar & 0.06 & 0.07 & 0.74 & -0.03 & 0.02 \\
\hline
\end{tabular}

Nota. Atitudes e curto prazo - Fator englobando as atitudes em relação à infidelidade e a estratégia sexual a curto prazo. Preocup. Preocupação. Motiv. - Motivação. Estr. - Estratégia.

O primeiro fator parece remeter para a qualidade e investimento na relação primária, estando associado de modo positivo à confiança, à paixão, a uma motivação autónoma para a fidelidade e a um investimento no parceiro a longo prazo, assim como a uma orientação política mais à direita. Está também associado, mas de modo negativo, ao conflito e à evitação. 0 segundo fator parece refletir sobretudo as atitudes em relação à infidelidade e a estratégia a curto prazo, estando associado a uma atitude positiva relativamente ao sexo casual e à infidelidade, assim como à utilização de uma estratégia de curto prazo. Parece estar também relacionado, de modo negativo, com a motivação controlada para a fidelidade, o que é coerente com a associação a atitudes contrárias a esta. 0 Fator 3 está relacionado com a perceção da qualidade das relações na família de origem (com a mãe e com o pai, entre os pais, qualidade da vida familiar e presença do pai). 0 Fator 4 parece remeter sobretudo para o estilo de vinculação preocupado, apresentando também associações com o investimento parental e no parceiro a longo prazo. 0 Fator 5 está associado à idade e à duração da relação primária.

Foram utilizados como preditores, nas análises de regressão subsequentes, resultados derivados para os fatores. Estes resultados agregam informação de todas as variáveis, ponderadas de acordo com a sua saturação no fator correspondente. Apresentam a importante vantagem de, para além de utilizarem o máximo de informação disponível, facilitarem a agregação de variáveis medidas em escalas muito diferentes. 0 seu processo de cálculo, no entanto, não possibilita o uso do coeficiente alfa de Cronbach.

\section{Preditores dos comportamentos extrarrelacionais no primeiro momento}

Efetuaram-se análises de regressão utilizando como variáveis dependentes os fatores da secção CD do QMCER, referentes à tipologia de comportamentos de infidelidade reportados pelos participantes, com o objetivo de perceber quais os melhores preditores destes comportamentos. A análise de regressão 
(Quadros 2 e 3) revelou, para o Envolvimento Sexual, uma capacidade de predição altamente significativa, quer para as mulheres, $F(5,244)=14.10 ; p<.001$, quer para os homens, $F(5,87)=9.72 ; p<.001$. A variância explicada é considerável, sendo maior para os homens (36 \%) do que para as mulheres (24\%). Tanto para as mulheres como para os homens, os únicos preditores significativos são os correspondentes ao investimento e qualidade na relação primária e ao uso de uma estratégia a curto prazo. Constata-se ainda que, entre as mulheres, estes dois fatores parecem dar um contributo equivalente enquanto, entre os homens, a maior orientação para uma estratégia a curto prazo parece ter mais peso do que o investimento na relação.

Quadro 2. Resultados das Análises de Regressão para as Mulheres no Primeiro Momento.

\begin{tabular}{|c|c|c|c|c|c|c|}
\hline & \multicolumn{2}{|c|}{ Envolv. Sexual } & \multicolumn{2}{|c|}{ Envolv. Afetivo } & \multicolumn{2}{|c|}{ Desejo Sexual } \\
\hline & $\boldsymbol{\beta}$ & $t$ & $\beta$ & $t$ & $\beta$ & $t$ \\
\hline Qualidade relação & -0.37 & $-6.60 * *$ & -0.40 & $-7.15^{* *}$ & -0.28 & $-5.19 * *$ \\
\hline Estr. Curto Prazo & 0.34 & $6.00 * *$ & 0.30 & $5.31^{* *}$ & 0.49 & $9.17^{* *}$ \\
\hline Família de Origem & 0.00 & 0.04 & -0.03 & -0.48 & -0.10 & -1.98 \\
\hline Preocupação & 0.03 & 0.60 & 0.08 & 1.45 & 0.07 & 1.28 \\
\hline Idade & 0.04 & 0.62 & 0.06 & 1.03 & 0.02 & 0.28 \\
\hline
\end{tabular}

Nota. Envolv. - Envolvimento.

${ }^{*} p<.05 .{ }^{* *} p<.01$.

Os resultados para o fator de Envolvimento Afetivo surgem semelhantes aos do anterior, mas com algumas diferenças que importa salientar. 0 modelo de regressão linear obtido é altamente significativo, tanto para as mulheres, $F(5,244)=15,29 ; p<.001$, como para os homens, $F(5,87)=4,49 ; p<.001$. A variância explicada é semelhante nos dois sexos, sendo de $24 \%$ para as mulheres e $21 \%$ para os homens.

Constata-se, tal como já tinha sido encontrado para o fator de Envolvimento Sexual, que os únicos preditores significativos, tanto entre os homens como entre as mulheres, são o investimento e qualidade na relação primária e o uso de uma estratégia a curto prazo, com atitudes favoráveis em relação à infidelidade. Verifica-se, no entanto, que, na predição do Envolvimento Afetivo, o investimento e qualidade na relação primária têm um peso relativamente maior do que na predição do Envolvimento Sexual. Assim, deixa de se verificar, entre os homens, uma preponderância das estratégias a curto prazo sobre a qualidade da relação primária, tendo agora os dois preditores igual peso. Pelo contrário, entre as mulheres, esse equilíbrio desfaz-se, passando a qualidade da relação primária a mostrar um peso mais acentuado como preditor.

Quadro 3. Resultados das Análises de Regressão para os Homens no Primeiro Momento.

\begin{tabular}{|c|c|c|c|c|c|c|}
\hline & \multicolumn{2}{|c|}{ Envolv. Sexual } & \multicolumn{2}{|c|}{ Envolv. Afetivo } & \multicolumn{2}{|c|}{ Desejo Sexual } \\
\hline & $\boldsymbol{\beta}$ & $t$ & $\beta$ & $t$ & $\beta$ & $t$ \\
\hline Qualidade relação & -0.18 & $-2.11^{*}$ & -0.31 & $-3.26^{* *}$ & -0.16 & -1.63 \\
\hline Estr. Curto Prazo & 0.56 & $6.48^{* *}$ & 0.29 & $2.99 * *$ & 0.41 & $4.28^{* *}$ \\
\hline Família de Origem & 0.04 & 0.41 & 0.00 & 0.03 & 0.10 & 1.07 \\
\hline Preocupação & 0.04 & 0.47 & 0.15 & 1.56 & 0.03 & 0.32 \\
\hline Idade & 0.14 & 1.57 & 0.09 & 0.89 & -0.00 & -0.03 \\
\hline
\end{tabular}

${ }^{*} p<.05 .{ }^{* *} p<.01$.

Para o fator de Desejo Sexual, o modelo de regressão linear surgiu de novo como altamente significativo, quer para as mulheres, $F(5,244)=22.66$; $p<.001$, quer para os homens, $F(5,87)=4.36$; $p<$ .001. A variância explicada foi de $32 \%$ para as mulheres e $20 \%$ para os homens. Os coeficientes de regressão permitem concluir que, em ambos os sexos, o uso de uma estratégia a curto prazo, com atitudes favoráveis em relação à infidelidade, constitui o preditor mais forte. Porém, enquanto nos homens este é o único preditor significativo, nas mulheres também uma baixa qualidade e investimento na relação primária e uma baixa qualidade da experiência de infância surgem como significativas.

\section{Preditores dos comportamentos extrarrelacionais no segundo momento}

Ao analisarmos os resultados referentes ao segundo momento de recolha de dados, o nosso objetivo foi o de investigar quais os preditores que permitem prever se aumentaram, diminuíram ou se se mantiveram os comportamentos de infidelidade reportados no primeiro momento, ou seja, prever qual o nível de envolvimento no segundo momento, controlando a mesma variável no primeiro momento. Para isso, foram de novo utilizados modelos de regressão linear (Quadro 4), mas desta vez num procedimento hierárquico em dois passos. No primeiro passo, foi introduzido como preditor o nível de envolvimento 
extrarrelacional no primeiro momento. No segundo, foram adicionados os cinco preditores já usados nas análises correspondentes ao primeiro momento, testando-se a significância da sua contribuição, em conjunto, para a predição das mudanças ocorridas no nível de envolvimento de um momento para o outro. Dado o número reduzido da amostra no segundo momento, nomeadamente no que concerne aos homens, não foi possível fazer a análise separadamente por sexos. No conjunto, porém, o número de casos disponíveis para esta análise, apesar de reduzido $(n=53)$, não é excessivamente problemático. Tendo em conta a magnitude dos efeitos esperados e aplicando os critérios propostos por Green (1991), verifica-se que este número de casos será suficiente para detetar correlações parciais entre 0.20 e 0.30 , o que nos parece adequado. Esta convicção foi confirmada pelos resultados encontrados, que não revelam problemas de poder de teste (os valores de $\beta$ a partir de 0.27 são significativos).

Quadro 4. Resultados das Análises de Regressão no Segundo Momento.

\begin{tabular}{|c|c|c|c|c|c|c|}
\hline & \multicolumn{2}{|c|}{ Envolv. Sexual } & \multicolumn{2}{|c|}{ Envolv. Afetivo } & \multicolumn{2}{|c|}{ Desejo Sexual } \\
\hline & $\beta$ & $t$ & $\beta$ & $t$ & $\beta$ & $t$ \\
\hline Momento 1 & 0.66 & $4.13^{* *}$ & 0.72 & $3.96^{* *}$ & 0.52 & $2.44^{*}$ \\
\hline Qualidade relação & -0.24 & -1.82 & 0.26 & 1.43 & -0.18 & -1.12 \\
\hline Estr. Curto Prazo & -0.06 & -0.40 & 0.08 & 0.51 & 0.23 & 1.04 \\
\hline Família de Origem & 0.03 & 0.24 & 0.51 & $3.87^{* *}$ & 0.18 & 1.28 \\
\hline Preocupação & -0.12 & -0.87 & 0.16 & 0.06 & 0.23 & 1.38 \\
\hline Idade & 0.27 & $2.23^{*}$ & -0.09 & -0.59 & 0.04 & 0.26 \\
\hline
\end{tabular}

${ }^{*} p<.05 .{ }^{* *} p<.01$.

Assim, relativamente ao fator de Envolvimento Sexual, verificou-se que a entrada dos preditores adicionais no segundo passo, apesar de permitir aumentar a percentagem de variância explicada, de $66 \%$ para $75 \%$, não atingiu a significância estatística, $F(5,22)=1.45 ; p=.25$. Ainda assim, e dado que a ausência de significância estatística poderá ser efeito da escassa dimensão da amostra, prosseguimos a análise, obtendo os coeficientes apresentados no Quadro 4. Constata-se que o único preditor significativo é o fator correspondente à idade, o que parece sugerir que as pessoas mais velhas e com relações primárias mais duradouras terão mais tendência para aumentar (em termos relativos) o seu nível de envolvimento sexual, do primeiro para o segundo momento de recolha de dados, enquanto as pessoas mais jovens e com relações de menor duração terão tendência para o diminuir. Embora não atingindo o valor convencional da significância estatística, o contributo do fator correspondente ao investimento e qualidade da relação aproxima-se desse valor e tê-lo-ia atingido, caso fosse usado um teste unilateral, o que até se justificaria neste caso. Assim, este resultado sugere que o investimento e qualidade da relação primária tende a fazer diminuir o envolvimento sexual.

Para o Fator de Envolvimento Afetivo, os preditores introduzidos no segundo momento permitem aumentar de modo significativo, de $36 \%$ para $67 \%$, a variância explicada, $F(5,22)=4.14 ; p=.01$, o que significa que, no seu conjunto, contribuem significativamente para explicar o aumento ou diminuição do envolvimento afetivo extrarrelacional. Examinando os preditores individualmente, o único que se mostra significativo é a perceção de qualidade na família de origem: os participantes que reportam uma maior qualidade na sua experiência de infância, mostram uma maior tendência para aumentar, do primeiro para o segundo momento de avaliação e em termos relativos, o seu nível de envolvimento emocional extrarrelacional.

Relativamente ao Fator de Desejo Sexual, o contributo dos fatores introduzidos no segundo momento voltou a não se mostrar significativo, $F(5,22)=0.95 ; p=.47$, o mesmo acontecendo para os preditores individuais.

\section{DISCUSSÃO}

Os resultados deste estudo afiguram-se como um avanço importante no estudo da infidelidade, dado que foi possível a identificação de preditores significativos dos comportamentos de infidelidade, quer no primeiro momento de recolha de dados, quer no segundo. 0 caráter longitudinal deste estudo constitui também uma mais-valia, pela escassez de estudos que avaliam esta temática em dois momentos no tempo e também porque foi possível identificar alguns preditores que parecem induzir mudanças nos comportamentos de infidelidade com o decorrer do tempo. Finalmente, o uso de um questionário que avalia separadamente as estratégias reprodutivas a curto e longo prazo ajuda a clarificar o contributo que este construto da psicologia evolutiva poderá trazer.

Relativamente à análise fatorial realizada aos preditores, apresentou a importante vantagem de reduzir o número de preditores e as suas intercorrelações, aumentando a validade das análises de regressão com um mínimo de perda da informação contida nas variáveis iniciais. Para além disso, 
permitiu clarificar a estrutura das variáveis presentes, associando, por exemplo, o estilo de vinculação evitante ao escasso investimento na relação primária e o estilo preocupado à estratégia a longo prazo, mas deixando claro que nenhum destes estilos de vinculação se relaciona de forma estreita com a estratégia reprodutiva a curto prazo ou com a qualidade da experiência de infância. Este resultado parece indicar que, ao contrário do defendido por alguns autores (e.g., Belsky, 1999), o contributo do estilo de vinculação evitante para o comportamento amoroso/sexual nos adultos não se confunde com o contributo das estratégias sexuais, enfatizadas pela psicologia evolutiva. Este resultado sugere, portanto, que estas duas teorias contribuem de forma paralela e independente para a predição dos comportamentos, neste caso, de infidelidade. Noutros casos, a análise indicou, pelo contrário, que as variáveis postuladas por diferentes teorias não cumprem critérios de validade discriminante, apresentando correlações entre si que permitem, em nome da parcimónia, reduzir drasticamente o número de preditores. É o caso das atitudes em relação ao sexo casual e à infidelidade, que se associam à estratégia reprodutiva a curto prazo (a primazia dada a uma ou outra variável na interpretação do fator tem, naturalmente, muito de arbitrário, mas a questão transcende o tema deste estudo). Esta análise terá, portanto, contribuído para amenizar uma certa proliferação teórica, visível na introdução deste artigo.

Em termos de preditores dos comportamentos de infidelidade, verificou-se um forte predomínio de dois deles: (a) a baixa qualidade e investimento na relação primária, associadas a um estilo de vinculação evitante, a uma maior motivação autónoma para a fidelidade e a uma orientação política mais conservadora, e (b) o uso de uma estratégia reprodutiva a curto prazo, associada a atitudes favoráveis em relação à infidelidade e à motivação controlada para a fidelidade. De facto, e com apenas uma exceção, estes surgem sempre como os mais fortes preditores, e os únicos significativos, das variadas facetas da infidelidade. Igualmente se confirma a ideia de que o primeiro estaria mais relacionado com o envolvimento afetivo e com o sexo feminino, e o segundo com o envolvimento sexual e o sexo masculino, embora estas diferenças apenas se verifiquem em termos de grau. De facto, embora estes preditores pareçam ter uma maior influência nos contextos apontados, ambos têm um efeito significativo na predição de ambos os tipos de envolvimento extrarrelacional e em ambos os sexos. Nomeadamente, parecem ter um peso quase igual na predição do envolvimento sexual por parte das mulheres e do envolvimento afetivo por parte dos homens. Apenas na predição do envolvimento afetivo por parte das mulheres e do envolvimento sexual por parte dos homens se verifica o predomínio de um deles, e ainda assim mantendo-se o outro como significativo. Em termos teóricos, estes resultados implicam que tanto a perspetiva da vinculação (Hazan \& Diamond, 2000; Hazan \& Shaver, 1994) como a da psicologia evolutiva (Buss, 2008; Buss e Schmitt, 1993) apresentam contributos relevantes para a explicação dos comportamentos extrarrelacionais. 0 papel de destaque encontrado para estas teorias contrasta com o de outras que, ou não surgiram de forma autónoma na análise fatorial dos preditores potenciais, ou pouca importância assumiram na predição dos comportamentos (caso da estratégia a longo prazo, associada ao estilo de vinculação preocupado).

Para além destes, apenas um outro preditor se revela significativo, e mesmo assim por estreita margem: uma experiência de infância mais negativa predispõe as mulheres para maior incidência de desejo sexual extrarelacional, mas esse desejo não parece depois traduzir-se em envolvimento sexual ou afetivo. Este resultado pode ser associado ao encontrado por Leal (2009), que encontrou um efeito direto da experiência de infância sobre o compromisso relacional, entre as mulheres apenas, e não mediado por variáveis da vinculação ou do investimento relacional. Parece assim confirmar-se um efeito de regulação do comportamento relacional adulto a partir da experiência de infância, mas limitado apenas às mulheres e com mecanismos de transmissão que investigações posteriores terão de ajudar a esclarecer. Este resultado parece, apesar de tudo, compatível com a formulação de Belsky (1999) que, apesar de mencionar o possível papel do estilo de vinculação como mediador, nunca comprovou empiricamente esse papel.

No que concerne às análises envolvendo o segundo momento de recolha de dados, importa sublinhar que não se apresentam como redundantes com as anteriores. Por outras palavras, os preditores da ocorrência de envolvimento não são os mesmos que permitem predizer a continuidade desse envolvimento passado um ano. A idade, associada a um maior tempo de relação, parece estar associada a um aumento relativo do envolvimento sexual do primeiro para o segundo momento. As possíveis explicações para este resultado não são evidentes, mas diversas hipóteses poderão ser levantadas. É possível que as pessoas mais velhas tenham tendência para envolvimentos sexuais mais duradouros, o que significaria que, quando esse envolvimento sexual surge, tende a aprofundar-se, enquanto nas pessoas mais jovens e com menor tempo de relação esses envolvimentos poderão ser mais transitórios e portanto não mostrar uma tendência clara.

Já o envolvimento afetivo parece ser sobretudo associado à família de origem, num efeito positivo, ou seja, quanto melhor a qualidade da relação na família de origem mais o envolvimento afetivo tenderá a 
manter-se ou a aumentar do primeiro para o segundo momento de recolha de dados. Mais uma vez, este efeito não se apresenta fácil de explicar, mas é plausível pensar que uma experiência de infância mais positiva leve a uma maior disponibilidade para o envolvimento emocional duradouro e que essa tendência se verifique mesmo em relações paralelas à principal.

Pensamos que a elevada escolaridade da amostra poderá ter influenciado os resultados, constituindo uma limitação, dado que há investigações que comprovam que esta variável tem influência nas práticas sexuais e no número de parceiros das mulheres (Alvarez \& Nogueira, 2008), e pode aumentar as oportunidades para o envolvimento em relações extraconjugais (Drigotas \& Barta, 2001). 0 facto de termos utilizado exclusivamente instrumentos de autorrelato também pode ter causado enviesamentos nos resultados, conduzindo a um decréscimo dos comportamentos de infidelidade assinalados, fruto do efeito de desejabilidade social. A elevada perda de participantes do primeiro para o segundo momento é também uma limitação importante, que condicionou muito as análises longitudinais realizadas. Outro aspeto tem a ver com a forma como foi operacionalizada a infidelidade no nosso estudo, baseando-se apenas na ocorrência de comportamentos previamente definidos, de envolvimento ou desejo sexual e afetivo, com pessoas que não o parceiro primário. Deste modo, não estão incluídas considerações, por exemplo, a respeito de existir ou não consentimento por parte do parceiro para esse envolvimento, ou sequer a opinião do autor ou autora dos comportamentos acerca de se eles constituem uma infidelidade. Espera-se que estudos futuros ajudem a esclarecer estes e outros aspetos, por exemplo recorrendo a uma metodologia mista, com métodos quantitativos e qualitativos, que permita uma maior exploração de uma variedade de outros tópicos.

\section{CONCLUSÃO}

Este estudo traz contributos relevantes para o estudo da infidelidade, ao colocar em confronto direto, no mesmo estudo, uma variedade de perspetivas teóricas, que muitas vezes se assumem como alternativas ou concorrentes na explicação dos comportamentos de infidelidade. A identificação da convergência de resultados entre algumas delas, mas também do contributo independente de outras, traz um enriquecimento do corpo teórico mas também da prática clínica, ao apontar o investimento e a qualidade da relação primária, assim como a utilização de uma estratégia de curto prazo, como principais preditores da infidelidade, atuando em simultâneo e de forma aditiva em diferentes contextos. 0 conhecimento destes preditores indicia ao terapeuta a necessidade de ter em conta ambos os tipos de variáveis, de forma independente, na compreensão e intervenção sobre o comportamento problemático, diferenciando o peso desta consideração em função do género do autor dos comportamentos e do tipo destes (envolvimento sexual e/ou afetivo), mas não deixando de ter em atenção os fatores que neste estudo surgiram como responsáveis pela manutenção ou aumento dos comportamentos (idade e duração da relação, para o envolvimento sexual, e experiências positivas na família de origem, para o envolvimento afetivo).

\section{Referências}

Afonso, C. (2011). Estilo de vinculação e relações extra-diádicas: Satisfação relacional e atitudes como mediadoras. Dissertação de Mestrado Integrado em Psicologia, Secção de Psicologia Clínica e da Saúde, Núcleo de Psicoterapia Cognitiva-Comportamental e Integrativa, Faculdade de Psicologia e de Ciências da Educação, Universidade de Lisboa.

Alvarez, M. J, \& Nogueira, J. (2008). Definições sexuais de estudantes universitários. Psicologia, 23, 59-76.

Barta, W. D., \& Kiene, M. (2005). Motivations for infidelity in heterosexual dating couples: The roles of gender, personality differences, and sociosexual orientation. Journal of Social and Personal Relationships 22, 339-360. doi:10.1177/0265407505052440

Belsky, J. (1999). Modern evolutionary theory and patterns of attachment. In J. Cassidy \& P. R. Shaver (Eds.), Handbook of Attachment (pp. 141-161). New York: Guilford.

Belsky, J., Steinberg, L., \& Draper, P. (1991). Childhood experience, interpersonal development, and reproductive strategy: An evolutionary theory of socialization. Child Development, 62, 647-670. doi:10.1111/j.1467-8624.1991.tb01558.x

Birnbaum, G., Reis, H., Mikulincer, M., Szepsenwol, O., Shaver, P., Mizrahi, M. (2014). When sex goes wrong: A behavioral systems perspective on individual differences in sexual attitudes, motives, feelings and behaviors. Journal of Personality and Social Psychology, 106, 822-842. doi:10.1037/a0036021

Brennan, K. A., Clark, C. L., \& Shaver, P. R. (1998). Self-report measurement of adult romantic attachment: An integrative overview. In J. A. Simpson \& W. Rholes (Eds.), Attachment theory and close relationships (pp. 46-76). New York: Guilford. 


\section{Mas Porquê? Um Estudo Multiteórico dos Preditores da Infidelidade}

Brooks, T., \& Monaco, K. (2013). Your cheatin' heart: Joint production, joint consumption and the likelihood of extramarital sex. Applied Economics Letters, 20, 272-275. doi:10.1080/13504851.2012.690845 Buss, D. M. (Ed.). (2005). The handbook of evolutionary psychology. New York: Wiley.

Buss, D. M. (2008). Evolutionary psychology: The new science of the mind. Boston, MA, USA: Pearson.

Buss, D. M., \& Schmitt, D. P. (1993). Sexual strategies theory: An evolutionary perspective on human mating. Psychological Review, 100, 204-232. doi:10.1037/0033-295X.100.2.204

Confer, J. C., Easton, J. A., Fleischman, D. S., Goetz, C. D., Lewis, D. G., Perilloux, C., \& Buss, D. M. (2010). Evolutionary psychology: Controversies, questions, prospects, and limitations. American Psychologist, 65, 110-126. doi:10.1037/a0018413

Deci, E., \& Ryan, R. (2008). Self-determination theory: A macrotheory of human motivation, development, and health. Canadian Psychology, 49, 182-185. doi:10.1037/a0012801

De Stefano, J., \& Oala, M. (2008). Extramarital affairs: Basic considerations and essential tasks in clinical work. The Family Journal: Counseling and Therapy for Couples and Families, 16, 13-19. doi:10.1177/1066480707309128

Draper, P., \& Harpending, H. (1982). Father absence and reproductive strategy: An evolutionary perspective. Journal of Anthropological Research, 38, 255-273.

Drigotas, S. M., \& Barta, W. (2001). The cheating heart: Scientific explorations of infidelity. Current Directions in Psychological Science, 10, 177-180. doi:10.1111/1467-8721.00143

Duba, J., Kindsvatter, A., \& Lara, T. (2008). Treating infidelity: Considering narratives of attachment. The Family Journal, 16, 293-299. doi:10.1177/1066480708323198

Eastwick, P. W., Luchies, L. B., Finkel, E. J., \& Hunt, L. L. (2014). The many voices of Darwin's descendants: Reply to Schmitt (2014). Psychological Bulletin, 140, 673-681. doi:10.1037/a0036111

Ellis, B. J., Bates, J. E., Dodge, K. A., Fergusson, D. M., Horwood, L. J., Pettit, G. S., \& Woodward, L. (2003). Does father absence place daughters at special risk for early sexual activity and teenage pregnancy? Child Development, 74, 801-821. doi:10.1111/1467-8624.00569

Ellis, B. J., \& Symons, D. (1990). Sex differences in sexual fantasy: An evolutionary psychology approach. Journal of Sex Research, 27, 527-555. doi:10.1080/00224499009551579

Elsmlie, B., \& Tebaldi, E. (2008). So, what did you do last night? The economics of infidelity. Kyklos, 61, 391-410. doi:10.1111/j.1467-6435.2008.00408.x

Fisher, H. E., Aron, A., Mashek, D., Li, H., \& Brown, L. L. (2002). Defining the brain systems of lust, romantic attraction, and attachment. Archives of Sexual Behavior, 31, 413-419. doi:10.1023/A:1019888024255

Foster, J., D., \& Misra, T., A. (2013). It did not mean anything (about me): Cognitive dissonance theory and the cognitive and affective consequences of romantic infidelity. Journal of Social and Personal Relationships, 30, 835-857. doi:10.1177/0265407512472324

Gaine, G., \& La Guardia, J. (2009). The unique contributions of motivations to maintain a relationship and motivations toward relational activities to relationship well-being. Motivation \& Emotion, 33, 184202. doi:10.1007/s11031-009-9120-x

Gilding, M. (2009). Paternity uncertainty and evolutionary psychology: How a seemingly capricious occurrence fails to follow laws of greater generality. Sociology, 43, 141-158. doi:10.1177/0038038508099102

Glass, S. P., \& Wright, T. L. (1992). Justifications for extramarital relationships: The association between attitudes, behaviors, and gender. Journal of Sex Research, 29, 361-387. doi:10.1080/00224499209551654

Goldenberg, M. (2008). Coroas: Corpo, envelhecimento, casamento e infidelidade. Rio de Janeiro: Record.

Goldenberg, M. (2013). Relações amorosas e conjugalidades: Amor, casamento e fidelidade na cultura brasileira. Gênero na Amazónia, 3, 13-24.

Green, S. B. (1991). How many subjects does it take to do a regression analysis? Multivariate Behavioral Research, 26, 499-510. doi:10.1207/s15327906mbr2603_7

Hall, J. H., \& Fincham, F. D. (2006). Relationship dissolution following infidelity: The roles of attributions and forgiveness. Journal of Social and Clinical Psychology, 25, 508-522. doi:10.1521/jscp.2006.25.5.508

Hazan, C., \& Diamond, L. (2000). The place of attachment in human mating. Review of General Psychology, 4, 186-204. doi:10.1037/1089-2680.4.2.186

Hazan, C., \& Shaver, P.R. (1994). Attachment as an organizational framework for research on close relationships. Psychological Inquiry, 5, 1-22. doi:10.1207/s15327965pli0501_1

Josephs, L. (2006). The impulse to infidelity and oedipal splitting. International Journal of Psychoanalysis, 87, 423-437. doi:10.1516/5A5V-WLPB-4HJ3-329J 
Kirkpatrick, L. A. (1998). Evolution, pair-bonding, and reproductive strategies: A reconceptualization of adult attachment. In J. A. Simpson \& W. S. Rholes (Eds.), Attachment theory and close relationships (pp. 353-393). New York: Guilford.

Klesse, C. (2006). Polyamory and its 'others': Contesting the terms of non-monogamy. Sexualities, 9, 565583. doi: $10.1177 / 1363460706069986$

Leal, A. R. P. (2009). De pequenino se torce o pepino: Processos de mediação entre as experiencias de infância e as relações de casal. Dissertação de Mestrado Integrado em Psicologia, Secção de Psicologia Clínica e da Saúde, Núcleo de Psicologia Clínica Dinâmica, Faculdade de Psicologia e de Ciências da Educação, Universidade de Lisboa.

Leak, G. K., \& Cooney, R. R. (2001). Self-determination, attachment styles, and well-being in adult romantic relationships. Representative Research in Social Psychology, 25, 55-62.

Lind, W. R. (2008). Casais biculturais e monoculturais: Diferenças e recursos. Dissertação de Doutoramento em Psicologia, Faculdade de Psicologia e de Ciências da Educação, Universidade de Lisboa.

Lopes dos Santos, P., Silva, A.P., \& Sousa, A. M. (1995). A escala Mother-Father- Peer. In L. S. Almeida, M. R. Simões, \& M. M. Gonçalves (Eds.), Provas Psicológicas em Portugal (pp. 257-269). Braga: APPORT.

Mikulincer, M., Florian, V., Cowan, P. A., \& Cowan, C. P. (2002). Attachment security in couple relationships: A systemic model and its implications for family dynamics. Family Process, 41, 405434. doi:10.1111/j.1545-5300.2002.41309.x

Mikulincer, M., \& Shaver, P. R. (2005). Attachment theory and emotions in close relationships: Exploring the attachment-related dynamics of emotional reactions to relational events. Personal Relationships, 12, 149-168. doi:10.1111/j.1350-4126.2005.00108.x

Moreira, J. M. (2004). Questionários: Teoria e prática. Coimbra: Almedina.

Moreira, J. M. (2009). Estilo de vinculação adulta como mediador da relação entre experiências parentais na infância e estratégias sexuais na idade adulta. Dados não publicados.

Moreira, J. M., Mata, A., Veríssimo, J., Mata, R. \& Moreira, S. (2003, Outubro). O que se faz por cá: Resultados Portugueses do "International Sexuality Description Project. Simpósio temático apresentado no V Simpósio Nacional de Investigação em Psicologia, Lisboa

Moreira, J. M., Lind, W., Santos, M. J., Moreira, A. R., Gomes, M. J., Justo, J., Oliveira, A. P., Filipe, L. A., \& Faustino, M. (2006). "Experiências em relações Próximas"; um questionário de avaliação das dimensões básicas dos estilos de vinculação nos adultos. Tradução e validação para a população portuguesa. Laboratório de Psicologia, 4, 3-27. doi:10.14417/lp.760

Pelletier, L., Tuson, K., Haddad, N. (1997). Client motivation for therapy scale: A measure of intrinsic motivation, extrinsic motivation and amotivation for therapy. Journal of Personality Assessment, 68, 414-435. doi:10.1207/s15327752jpa6802_11

Poulsen, F., Holman, T., Busby, D. Carroll, J. (2013). Physical attraction, attachment styles and dating development. Journal of Social and Personal Relationships, 30, 301-319. doi:10.1177/0265407512456673

Schmitt, D. P. (2005a). Is short term mating the maladaptive result of insecure attachment? A test of competing evolutionary perspectives. Personality and Social Bulletin, 20, 1-23. doi:10.1177/0146167204271843

Schmitt, D. P. (2005b). Sociosexuality from Argentina to Zimbabwe: A 48-nation study of sex, culture and strategies of human mating. Behavioral and Brain Sciences, 28, 247-311. doi:10.1017/S0140525X05000051

Simpson, J. A., \& Gangestad, S. W. (1991). Individual differences in sociosexuality: Evidence for convergent and discriminant validity. Journal of Personality and Social Psychology, 60, 870-883. doi:10.1037/0022-3514.60.6.870

Smiler, A. P. (2011). Sexual strategies theory: Built for the short term or the long term? Sex Roles, 64, 603612. doi:10.1007/s11199-010-9817-z

Stefanou, C., \& McCabe, M. P. (2012). Adult attachment and sexual functioning: A review of past research. Journal of Sexual Medicine, 9, 2499-2507. doi:10.1111/j.1743-6109.2012.02843.x

Treas, J., \& Giesen, D., (2000). Sexual infidelity among married and cohabiting Americans. Journal of Marriage and Family, 62, 48-60. doi:10.1111/j.1741-3737.2000.00048.x

Viegas, T., \& Moreira, J. M. (no prelo). Os sete lados do triângulo: Desenvolvimento do Questionário Multidimensional de Comportamentos Extrarrelacionais (QMCER). Revista Iberoamericana de Diagnóstico e Avaliação Psicológica.

Vigil, J. M., \& Geary, D. C. (2006). Parenting and community background and variation in women's lifehistory development. Journal of Family Psychology, 20, 597-604. doi:10.1037/0893-3200.20.4.597 
Wilson, K., Mattingly, B., Clark, E., Weidler, D., Bequette, A. (2011). The gray area: Exploring attitudes toward infidelity and the development of the perceptions of dating infidelity scale. The Journal of Social Psychology, 151, 63-86. doi:10.1080/00224540903366750

Zeifman, D., \& Hazan, C. (1997). A process model of adult attachment formation. In S. Duck (Ed), Handbook of personal relationships (2nd ed., pp. 17-195). Chichester, England: John Wiley \& Sons.

\section{Historial do artigo \\ Recebido 31/12/2014 \\ Aceite $\quad 14 / 07 / 2015$ \\ Publicado 12/2015}

Apoio à publicação: Fundação para a Ciência e a Tecnologia (Ministério da Educação e Ciência, Portugal) - Programa FACC 